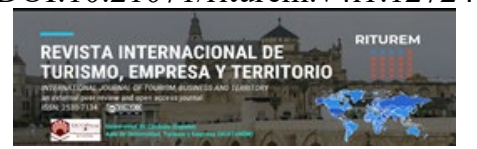

Cita bibliográfica: Doumet Chilán, N.Y. (2020). Fundamentos teóricos para una estrategia de conservación y valorización turístico-recreativa de los humedales de Ecuador. Revista Internacional de Turismo, Empresa y Territorio, 4 (1), $42-60$. https://doi.org/10.21071/riturem.v4i1.12724

\title{
Fundamentos teóricos para una estrategia de conservación y valorización turístico-recreativa de los humedales de Ecuador
}

\author{
Theoretical foundations for a conservation strategy for wetlands in Ecuador
}

\author{
Neme Yamil Doumet Chilán ${ }^{1 *}$
}

\begin{abstract}
Resumen
Este trabajo tiene como objetivo central identificar las fundamentaciones teóricas y bases científicas de la planificación y manejo sostenible de los humedales de Ecuador, partiendo de la situación y significación actual de sus ecosistemas y sus problemáticas socioambientales. En primer lugar, se estudian las causas y factores desencadenantes de su deterioro y pérdida de biodiversidad, como los intereses económicos particulares, la escasa sensibilidad y el desconocimiento de su importancia ambiental por parte de los gestores locales y la poca valoración social que aún tienen en muchos territorios. La metodología empleada ha partido de un enfoque cualitativo y en una revisión sistemática de la bibliografía sobre el tema a nivel internacional, así como de información de tipo técnico y y documentación oficial relativa a las políticas públicas con incidencia en estos espacios protegidos. Posteriormente se define un marco teórico para el manejo integral de los humedales, considerando también la significación de las actividades turístico-recreativas como herramienta de valorización, gestión y manejo sostenible de estos espacios. Asimismo, se ha establecido una matriz de conceptualización en la que se acotan las principales definiciones y términos sobre los humedales en Ecuador. Para finalizar se analiza la clasificación de estos espacios desde la perspectiva de sus características biológicas y físicas según los lineamientos de la Secretaría de la Convención Ramsar, que establece un total de 30 categorías de humedales naturales y otras 9 de humedales artificiales.
\end{abstract}

Palabras clave: Turismo; Áreas protegidas; Humedales; Desarrollo sostenible; Marco teórico; Ecuador.

\begin{abstract}
The objective of this work was to support the conceptualization of the sustainable management of wetlands in Ecuador. The aim was to establish the theoretical-scientific bases on these ecosystems considering their characteristics and socio-environmental problems. There are several causes of its deterioration, among them private economic interests, ignorance on the part of local managers and low social valuation. The methodology used followed a qualitative approach based on the bibliographic review, search for technical information and official documents. Research found in specialized scientific literature was taken as a reference. Then a theoretical framework for the comprehensive management of wetlands was proposed, considering the tourist activity as a management and conservation tool. A conceptualization matrix was established where the main definitions of wetlands in Ecuador are described. Finally, the classification is analyzed according to the biological and physical characteristics
\end{abstract}

\footnotetext{
${ }^{1}$ Escuela Superior Politécnica Agropecuaria de Manabí (ESPAM MFL), Ecuador. E-mail de contacto: doumetour@hotmail.com. Id orcid: 0000-0003-4295-5270 * Autor para la correspondencia.
} 
according to the guidelines of the Secretariat of the Ramsar Convention, which classifies them into 30 categories of natural and 9 artificial wetlands.

Keywords: Tourism; Protected areas; Wetlands; Sustainable development; Theoretical framework; Ecuador.

\section{Introducción}

Numerosos autores como Polk et al., (2017) sostienen con propiedad que los humedales tienen un valor ecológico muy alto en lo que respecta a la conservación de la biodiversidad y otras variables como la cobertura del suelo, el equilibrio climático y el flujo hídrico sostenido. Por otro lado, si se analiza desde diferentes puntos de vista la importancia de estos sistemas acuáticos podemos convenir en que su importancia no solo radica en su significación ambiental, sino que, como señalan Doumet y Rivera (2018), también se deben considerar los servicios ecosistémicos que estos humedales prestan al bienestar social de las comunidades locales aledañas y sus múltiples beneficios y recursos que facilitan a éstas (agua, alimento, regulación climática, generación de empleo, medicina, recreación...). Pero esto lamentablemente no quita para que estos valores no sean suficientemente conocidos y que, como consecuencia, estas áreas naturales estén sufriendo un progresivo deterioro. En este sentido, Borja et al. (2012) señalan cómo muchos de ellos han pasado de ser sistemas naturales, con distinto nivel de conservación, a sistemas ecológico-económicos que han servido de soporte a usos y actividades tradicionales como la caza, la pesca, la extracción de sal, la agricultura o la regulación hídrica.

En cualquier caso, estamos hablando de territorios en donde el agua es el elemento fundamental, ya que interactúa de forma continua en todos sus procesos ecosistémicos y en los diferentes hábitats, formando áreas con elevados índices de biodiversidad. Por ello, Xiaofei et al., (2018) afirman que los humedales son hoy día ecosistemas muy importantes para el desarrollo sostenible en los que se pueden compatibilizar, con una buena planificación y manejo sostenible de los recursos hídricos, actividades como la agricultura y el uso del agua para el abastecimiento de las poblaciones; de aquí el interés a escala mundial por su conservación. Pero si no se siguen modelos integrales que busquen la gestión ambiental eficiente de estos ecosistemas muchos serían los resultados negativos y muchos también los perjudicados en las comunidades locales que se asientan en los alrededores de estas áreas.

Para el manejo sostenible de cualquier ecosistema es importante señalar que la planificación tiene que ser considerada desde una perspectiva integral y multifacética que tenga en cuenta las sinergias favorables que pueden establecerse entre el turismo y la recreación, como instrumentos de generación de ingresos, y la conservación y la gestión ambiental, que pueden financiarse al menos parcialmente a través de esas inyecciones económicas, sobre todo en los territorios con mayores índices de biodiversidad y de fragilidad ambiental y con prioridades de sostenibilidad (Ooi, Duke y O'Leary, 2018). El manejo sostenible, en fin, contribuye a la conservación de las especies, el fortalecimiento de manifestaciones y prácticas socioculturales muy ligadas a estos humedales y la diversificación de las economías locales. Y es aquí donde se puede evidenciar el interés del ecoturismo como instrumento de desarrollo y práctica responsable para estos humedales al tiempo que como activo económico y de educación ambiental (Mulero y Rivera, 2018).

De Groot et al. (2010) explican, asimismo, que el valor que poseen los humedales es muy diverso y se refleja en diversos ámbitos territoriales (ambiental, social, cultural, económico y recreativo); de aquí que haya de buscarse un equilibrio, aunque sea ciertamente difícil, entre estas distintas vertientes consideradas mediante la planificación y la definición de políticas públicas 
que regulen y orden los distintos usos del suelo en el contexto de la sostenibilidad integral del ecosistema. En relación con todo esto, Orgaz (2014) manifiesta que el desarrollo del ecoturismo en los humedales exige de la implicación, colaboración y corresponsabilidad de los stakeholders implicados en esta actividad turística, pero para ello ha de planificarse adecuadamente y conseguir en último término el objetivo de que los beneficios de dicha actividad se concentren en su mayor parte en las comunidades locales del área de influencia de estos espacios, de manera compatible con la conservación de los humedales y la minimización de los impactos territoriales negativos.

Lo cierto es que muchos humedales cuentan con suficientes recursos y entidad territorial como para albergar actividades tan diversas como la agricultura y el turismo, que en realidad pueden no solo compatibilizarse sino también conjugarse de forma sostenible para dar lugar a productos de agroecoturismo (Park et al., 2017). Otra cosa, eso sí, es que lamentablemente algunos gestores no posean una visión holística y manejen de forma separada e inadecuada, como si se trataran de compartimentos estancos, las distintas piezas territoriales y usos del suelo de las zonas húmedas. Y, por otra parte, hay que considerar, como lo hace la Secretaría de la Convención Ramsar (2016), que una parte importante de los destinos turísticos más apreciados y desarrollados a nivel mundial se ubican en áreas insulares, costeras y litorales que cuentan con ecosistemas de humedales que frecuentemente se conforman como un recurso territorial y un factor de atracción ciertamente importantes para los visitantes que se acercan a dichos destinos. Sin embargo, se tienen que tomar medidas como el control de visitantes, además de maximizar los beneficios que el turismo pueda aportar para que reviertan tanto para la conservación como para el desarrollo local.

Según la Secretaría de la Convención Ramsar (2013) los planes de manejo para los humedales son herramientas esenciales para resolver los múltiples problemas derivados de las actividades económicas que en ellos se desarrollan, incluida la actividad turística. La planificación debe integrarse con la gestión de ecosistemas vinculando los objetivos loables de conservación a la realidad sociocultural de cada territorio. Reafirmando lo dicho, Woodcock (2010) sostiene que el planeamiento, así como las políticas nacionales, regionales y locales específicas, son factores fundamentales para la conservación del ambiente y el aseguramiento del desarrollo inclusivo de la economía local en las áreas de influencia socioeconómica de los humedales, tomando en cuenta en los planes de manejo los aspectos intangibles que son la base de una cultura o comunidad y también, como indican Minoo y Albrecht (2016), el patrimonio cultural en su conjunto.

Hay que tener, en definitiva, una visión amplia de la importancia que tienen estos biomas para las sociedades contemporáneas considerando todos los factores y variables que los caracterizan, pues de lo contrario su desconocimiento o tratamiento sesgado y parcial sólo conllevaría a su deterioro o incluso su desaparición. Como señalan Martina et al. (2019), los humedales constituyen zonas que puedan aportar importantes beneficios económicos, si bien éstos no son tan visibles por parte de los actores locales por una falta de conocimiento técnico de estas potencialidades y de los valores de los humedales como corredores ambientales, reguladores climáticos, generadores de biodiversidad y concentración de recursos hídricos que puedan resultar básicos para sectores productivos como la agricultura, la pesca, la caza y la ganadería y la actividad turístico-recreativa.

Mora y Ramírez (2019) sostienen que el turismo de actividades blandas y sostenibles en el medio natural como el aviturismo perfectamente pueden convertirse en una alternativa económica viable y compatible con la conservación de los humedales, ya que su impacto es muy inferior al otras actividades más intensivas e invasivas. A nivel mundial estos ecosistemas cumplen un papel ecológico importante al ser fuente de alimento, anidación y abrigo para la avifauna residente y migratoria, por lo que estos humedales suelen tener una gran concentración de avifauna, 
convirtiéndose ésta en un atractivo singular que suele interesar a un público aún un tanto minoritario pero con una capacidad adquisitiva y de generación de ingresos nada desdeñable y, sobre todo, con gran sensibilidad y respeto hacia el medio natural que visitan y las comunidades locales aledañas. Esos territorios, eso sí, para captar esta demanda de turismo ornitológico, han de contar con un plan de control y manejo del espacio natural, un estudio de capacidad de carga turístico-recreativa y equipamientos específicos con facilidades y servicios para los visitantes y turistas especializados en esta actividad tales como miradores, puestos de avistamiento de aves, centros de interpretación, hitos de educación ambiental, etc. (Rivera, 2010).

Considerando el país que estudiamos en este trabajo (Ecuador), Echevarría (2008) resalta la existencia en el mismo de una gran biodiversidad que ha dado lugar a la identificación a lo largo y ancho de su geografía de diferentes tipos de humedales según su localización geográfica y características ecológicas y territoriales. Se pueden distinguir, en este sentido, ecosistemas marinos-costeros en las áreas del Pacífico y región insular de las islas Galápagos, ecosistemas de bosque húmedo y seco tropical en regiones del interior de la costa $\mathrm{y}$, asimismo, espacios continentales de páramos y alta montaña en la cordillera de los Andes y la región Amazónica. No obstante, como señala Spence (2011), la delimitación y caracterización de estos sistemas ecológicos a nivel mundial y regional es bastante compleja y aún se deben de abordar no pocos estudios exhaustivos sobre su situación actual, funciones ecológicas, problemas ambientales y sostenibilidad y potencialidades de desarrollo, con el objeto de poder tomar decisiones futuras para su correcta gestión y uso sostenible. Partiendo de estas consideraciones es un hecho innegable la necesidad de fortalecer las acciones e investigaciones que promuevan el estudio de sus tipologías, problemáticas y características, así como la conservación de los humedales y el desarrollo sostenible de sus entornos de influencia en función de sus especificidades ecológicas y socioculturales.

El Ministerio del Ambiente de Ecuador (MAE, 2013) tiene bajo su responsabilidad las políticas de conservación de un total de 19 humedales protegidos por el Convenio Ramsar, que comprenden una superficie total de 290.815 hectáreas. El país suscribió los acuerdos de la Convención Ramsar en 1990, entrando en vigor plenamente el 7 de enero de 1991, lo que dio lugar a la realización de esfuerzos importantes para dar cumplimiento a los compromisos de conservación y uso racional de los humedales previstos en dicha Convención. Pese a esto ya se alertó de las problemáticas preexistentes que debían de reconducirse y minimizarse, entre ellas: degradación y pérdida acelerada de hábitats por presiones antrópicas, tendencia a la desecación, reducción de espejos de agua, eutrofización, uso inadecuado de los recursos hídricos superficiales por ampliación de la frontera agrícola y la construcción de infraestructuras como presas hidroeléctricas y construcción de carreteras.

Aunque se reconozca en términos generales la importancia de la biodiversidad en Ecuador, lo cierto es que en algunas regiones del país no está lo suficientemente potenciada y valorizada, como es el caso de los ecosistemas de humedales que forman parte de esa extraordinaria megadiversidad, a lo que se suma el hecho de que, como señalan Llambí y Cuesta (2014), existen zonas como los Andes ecuatorianos donde se han estudiado relativamente poco los humedales (el páramo y su ecología, por ejemplo, han sido mucho menos estudiados que la vegetación y las comunidades de plantas vasculares). Por tanto, queda aún una tarea importante de estudio y conocimiento de estos ecosistemas y sus casuísticas territoriales diversas, así como de difusión y valorización de los servicios ambientales y ecosistémicos que brindan a la sociedad. En el caso de los humedales altoandinos que forman parte de los ecosistemas de páramo, por ejemplo, la misma Convención de Ramsar y el Grupo de Contacto EHAA (2008) defienden que no deben ser tratados como cuerpos de agua aislados, sino como complejos o sistemas y, en consecuencia, se incluyen sus microcuencas de captación, y en los mismo hay que considerar de manera integrada 
y sistémica las interrelaciones sociales, económicas, ecológicas y culturales que se manifiestan en estos ecosistemas.

\section{Metodología}

El objetivo de este trabajo es analizar e identificar un marco teórico que sirva como fundamento conceptual para fundamentar proyectos y decisiones direccionadas a la conservación y uso sostenible de los humedales en Ecuador. Para ello se ha realizado una búsqueda y sistematización bibliográfica especializada en el estudio de los humedales y sus funciones en el ciclo hidrológico, ecológico y los múltiples beneficios socioeconómicos que pueden aportar a las comunidades locales.

A partir de aquí, se ha planteado una fundamentación científico-conceptual sobre la definición, caracterización, clasificación y problemáticas básicas de los humedales en Ecuador. Y particularmente se han tomado como referencias las investigaciones de Nunes, Fernández y Junk (2014) sobre clasificación de Áreas Húmedas de Brasil, además de algunos trabajos del Ministerio del Ambiente de Ecuador (MAE) y fuentes científicas de la Secretaría de la Convención Ramsar.

El procedimiento metodológico de esta investigación utiliza un enfoque cualitativo y aplica técnicas de investigación bibliográfica a nivel exploratorio-descriptivo. Y finalmente se plantea un marco teórico para el manejo integral y sostenible de los humedales ecuatorianos considerando a la actividad turística como una herramienta válida para la valorización, conservación y gestión sostenible de estas áreas de importancia ambiental. Para alcanzar los objetivos planteados se presenta el siguiente marco metodológico de la Tabla 1 siguiente:

Tabla 1. Marco metodológico

\begin{tabular}{|c|c|c|}
\hline FASES & ACTIVIDADES & $\begin{array}{l}\text { TÉCNICAS, } \\
\text { HERRAMIENTAS }\end{array}$ \\
\hline $\begin{array}{l}\text { Análisis del marco teórico, } \\
\text { conceptual y territorial de } \\
\text { los humedales ecuatorianos }\end{array}$ & $\begin{array}{l}\text { 1-Levantamiento de información } \\
\text { bibliográfica en fuentes } \\
\text { especializadas y científicas. } \\
\text { 2-Estructuración del marco } \\
\text { conceptual y teórico. } \\
\text { 3Análisis comparativo de la } \\
\text { clasificación de los humedales } \\
\text { según Ramsar Ecuador. } \\
\text { 4-Definición de los diferentes tipos } \\
\text { de humedales de acuerdo con sus } \\
\text { regiones geográficas de ubicación y } \\
\text { características físico-territoriales. }\end{array}$ & $\begin{array}{l}\text { Método analítico y sintético. } \\
\text { Fichas de registro. Lista de } \\
\text { chequeo. Análisis de } \\
\text { documentos. Revisión } \\
\text { bibliográfica. Comparación } \\
\text { histórico-lógica. }\end{array}$ \\
\hline
\end{tabular}

Fuente: Elaboración propia

Con la revisión y búsqueda de fuentes bibliográficas y documentales sobre los humedales a nivel internacional y nacional, se han podido analizan conceptos teóricos y metodológicos en lo que concierne a estos ecosistemas acuáticos. Se han utilizado, en este sentido también, tanto 
fuentes primarias como secundarias, consultado diversas bases de datos y repositorios de revistas científicas. También se ha buscado información en portales oficiales, repositorios de información y documentos oficiales de organizaciones vinculadas con el manejo y conservación de los humedales (Ministerio del Ambiente de Ecuador (MAE), Ministerio de Turismo (MINTUR), Secretaria Convención Ramsar, Gobiernos provinciales y locales, ONG, etc.). Las fuentes consultadas se han organizado finalmente mediante una ficha de registro y una lista de chequeo.

Una vez identificada y sistematizada toda esta información se han obtenido y estructurado los datos más relevantes y también se ha acometido un análisis comparativo de las definiciones empleadas según regiones y países. Posteriormente, se ha planteado una matriz de síntesis mostrando los conceptos que son la base para el marco teórico, además de proceder a la clasificación de los humedales identificados de acuerdo con sus características biológicas y físicas y los lineamientos de la Secretaría de la Convención Ramsar, la cual los clasifica en 30 categorías de humedales naturales y 9 artificiales. Además, se ha organizado una tabla con los diferentes tipos y nombres de humedales que son más conocidos en cada una de las regiones geográficas de Ecuador.

\section{Resultados y discusión}

En la Tabla 2 siguiente hemos procedido a representar una matriz con las definiciones y la terminología más utilizadas y comúnmente aceptadas sobre los humedales y sus ecosistemas, tanto por parte de organismos oficiales nacionales e internacionales como en la bibliografía científica especializada.

En esta matriz se han podido identificar, por ejemplo, las causas más frecuentes que han dado lugar a la desaparición de los humedales, como es el caso del desconocimiento de su importancia y valor ecosistémico y sociocultural por parte de los gestores, actores locales y representantes de las sociedades que viven en sus entornos territoriales más próximos. Y en el caso de Ecuador, en particular, se han podido evidenciar estas problemáticas especialmente en la región costero-litoral por la cercanía a zonas productivas tanto de acuicultura, agricultura y ganadería.

Tabla 2. Definiciones y terminología sobre humedales

\begin{tabular}{|c|c|}
\hline AUTOR & DEFINICIÓN \\
\hline $\begin{array}{lr}\text { Asamblea } & \text { Nacional } \\
\text { Constituyente del } & \text { Ecuador. } \\
(2008) \text { Art. } & 406 . \\
\text { Constitución de la República } \\
\text { del Ecuador del } 20 \text { de octubre } \\
\text { de } 2008\end{array}$ & $\begin{array}{l}\text { "El Estado regulará la conservación, manejo y uso sustentable, } \\
\text { recuperación, y limitaciones de dominio de los ecosistemas frágiles } \\
\text { y amenazados; entre otros, los páramos, humedales, bosques } \\
\text { nublados, bosques tropicales secos y húmedos y manglares, } \\
\text { ecosistemas marinos y marinos-costeros." }\end{array}$ \\
\hline $\begin{array}{lr}\text { Asamblea } & \text { Nacional } \\
\text { Constituyente del } & \text { Ecuador. } \\
(2008) \text { Art. } & 411 .- \\
\text { Constitución de la República } \\
\text { del Ecuador del } 20 \text { de octubre } \\
\text { de } 2008\end{array}$ & $\begin{array}{l}\text { El Estado garantizará la conservación, recuperación y manejo } \\
\text { integral de los recursos hídricos, cuencas hidrográficas y caudales } \\
\text { ecológicos asociados al ciclo hidrológico. La sustentabilidad de los } \\
\text { ecosistemas y el consumo humano serán prioritarios en el uso y } \\
\text { aprovechamiento del agua." }\end{array}$ \\
\hline $\begin{array}{l}\text { Art. 20.- Libro V: De la } \\
\text { Gestión de los Recursos } \\
\text { Costeros }\end{array}$ & $\begin{array}{l}\text { Se declaran bosques protectores a los manglares existentes en las } \\
\text { provincias de Esmeraldas, Manabí, Guayas y El Oro que fueran de } \\
\text { dominio del Estado. Con la participación del Ministerio de Defensa, }\end{array}$ \\
\hline
\end{tabular}




\begin{tabular}{|c|c|}
\hline & $\begin{array}{l}\text { Consejo Nacional de Recursos Hídricos y Corporaciones de } \\
\text { Desarrollo Regional de acuerdo con el Art. } 6 \text { de la mencionada Ley } \\
\text { Forestal." }\end{array}$ \\
\hline $\begin{array}{lcr}\text { Artículo } 40 & \text { Ley } & \text { para la } \\
\text { Conservación } & \text { y } & \text { Uso } \\
\text { sustentable } & \text { de } & \text { la } \\
\text { Biodiversidad de } & 2004 & \end{array}$ & $\begin{array}{l}\text { Los ecosistemas frágiles son aquellos que, por sus condiciones } \\
\text { biofísicas, culturales, nivel de amenaza o por interés público, deben } \\
\text { ser objeto de un manejo particularizado y son declarados como tales } \\
\text { por el Ministerio del Ambiente, de oficio o a petición de parte } \\
\text { interesada: a) Manglares y otros humedales establecidos como tales } \\
\text { en la Convención de RAMSAR; b) Páramos; y, c) Bosques secos, } \\
\text { bosques nublados y de garúa. }\end{array}$ \\
\hline $\begin{array}{lrr}\text { Artículo 43.- } & \text { Ley } & \text { para la } \\
\text { Conservación } & \text { y } & \text { Uso } \\
\text { sustentable } & \text { de } & \text { la } \\
\text { Biodiversidad de } & 2004 & \end{array}$ & $\begin{array}{l}\text { El Estado normará y promoverá la conservación y uso sustentable de } \\
\text { los humedales que sean declarados ecosistemas frágiles, de } \\
\text { conformidad con lo dispuesto por esta Ley, su reglamento y el } \\
\text { correspondiente Plan de Manejo que deberá ser aprobado y } \\
\text { periódicamente supervisado por el Ministerio del Ambiente y de } \\
\text { conformidad con los principios de la Convención de Ramsar. La } \\
\text { conservación de los humedales de agua dulce estará integrado al } \\
\text { manejo y gestión de las cuencas hidrográficas del país. }\end{array}$ \\
\hline $\begin{array}{l}\text { Artículo 34. Ley de Recursos } \\
\text { Hídricos, Usos } \\
\text { Aprovechamiento del Agua } \\
\text { de } 2014\end{array}$ & $\begin{array}{l}\text { Gestión integrada e integral de los recursos hídricos. La Autoridad } \\
\text { Única del Agua es responsable de la gestión integrada e integral de } \\
\text { los recursos hídricos con un enfoque ecosistémico, Conservar y } \\
\text { manejar sustentablemente los ecosistemas marino-costeros, } \\
\text { altoandinos y amazónicos, en especial páramos, humedales y todos } \\
\text { los ecosistemas que almacenan agua. }\end{array}$ \\
\hline $\begin{array}{l}\text { La Secretaría de la } \\
\text { Convención Ramsar }(2010)\end{array}$ & $\begin{array}{l}\text { Extensiones de marismas, pantanos y/o turberas, superficies éstas } \\
\text { cubiertas de aguas, sean éstas de régimen natural o artificial, } \\
\text { permanentes o temporales, estancadas o corrientes, dulces, salobres } \\
\text { o saladas, incluidas las extensiones de agua marina cuya profundidad } \\
\text { en marea baja no exceda de seis metros. Los humedales se dan donde } \\
\text { la capa freática se localiza al nivel de la superficie terrestre o cerca } \\
\text { de ella o donde la tierra está cubierta por aguas poco profundas, de } \\
\text { manera que el agua es el principal factor controlador del medio y la } \\
\text { vida vegetal y animal asociada a él. }\end{array}$ \\
\hline $\begin{array}{l}\text { Nunes, Fernández y Junk } \\
(2014)\end{array}$ & $\begin{array}{l}\text { Ecosistemas de integración entre ambientes terrestres y acuáticos, } \\
\text { continentales o costeros, naturales y artificiales, permanentemente } \\
\text { inundados por aguas o de manera temporal en rasas o suelos } \\
\text { encharcados, con agua dulce o salobre, que sirven de hábitat a } \\
\text { numerosas comunidades de plantas y animales adaptados a su } \\
\text { dinámica hídrica }\end{array}$ \\
\hline Martín et al., (2010) & $\begin{array}{l}\text { Ecosistemas con la mayor biodiversidad del planeta y a la vez, los } \\
\text { más amenazados por la sobreexplotación y el uso irracional de sus } \\
\text { recursos, especialmente importantes por el papel que juegan en el } \\
\text { mantenimiento de los valores culturales asociados al uso de sus } \\
\text { recursos territoriales como la pesca artesanal. }\end{array}$ \\
\hline Cole. (2017) & $\begin{array}{l}\text { Son territorios en donde el recurso hídrico es el elemento } \\
\text { fundamental, ya que interactúa de forma continua en todos sus } \\
\text { procesos ecosistémicos y en los diferentes hábitats, formando áreas } \\
\text { con elevados índices de biodiversidad, prestan diversos servicios } \\
\text { vitales para los habitantes de su entorno regional y contribuyen al } \\
\text { desarrollo socioeconómico y al buen vivir de las comunidades locales }\end{array}$ \\
\hline
\end{tabular}




\begin{tabular}{|c|c|}
\hline $\begin{array}{l}\text { Do, Y., Kim, S.-B., Kim, J. } \\
\text { Y., \& Joo, G.-J. (2015). }\end{array}$ & $\begin{array}{l}\text { Los humedales no son exclusivamente terrestres o acuáticos, sino que } \\
\text { más bien pueden ser ambos simultáneamente, como ecosistemas } \\
\text { temporales o permanentes que influyen directamente en el proceso } \\
\text { sistémico de la fauna y la flora. }\end{array}$ \\
\hline $\begin{array}{l}\text { Chaoyang Fang, Zhanghua } \\
\text { Tao, Dan Gao \& Hao Wu } \\
(2016)\end{array}$ & $\begin{array}{l}\text { Son zonas de transición entre ecosistemas terrestres y acuáticos que } \\
\text { juegan un papel vital en la estabilidad ambiental, purificación del } \\
\text { agua, preservación de la biodiversidad y control de la erosión e } \\
\text { inundaciones. }\end{array}$ \\
\hline $\begin{array}{l}\text { Songjun Xu, Hyun Jeong } \\
\text { Kim, Mingzhu Liang \& } \\
\text { Kisang Ryu (2018) }\end{array}$ & $\begin{array}{l}\text { El Humedal es uno de los tres principales ecosistemas en el planeta } \\
\text { y puede ser encontrado entre los bosques y océanos en la tierra, se } \\
\text { considera como "el riñón de la tierra". Desempeña un papel } \\
\text { importante no solo en el servicio del ecosistema, sino también como } \\
\text { soporte y cualificación de actividades turísticas y recreativas. }\end{array}$ \\
\hline Alfonso y Dipotet. (2009). & $\begin{array}{l}\text { Los humedales comprenden entre el } 4 \text { y el } 6 \% \text { de la superficie } \\
\text { terrestre. La presencia de agua el principal elemento para el } \\
\text { ecosistema, que puede ser temporal o permanente, lo que motiva el } \\
\text { desarrollo característico de suelos, microorganismos y comunidades } \\
\text { de plantas y animales, de modo que funcionen en forma diferente y } \\
\text { especializada. }\end{array}$ \\
\hline Keddy, (2004). & $\begin{array}{l}\text { Los humedales como marismas, pantanos o ciénegas son ecosistemas } \\
\text { que surgen cuando la saturación del terreno por agua produce } \\
\text { sustratos hídricos (dominados por procesos anaeróbicos) y permite el } \\
\text { establecimiento de biota, principalmente plantas enraizadas, con } \\
\text { adaptaciones para tolerar la anegación }\end{array}$ \\
\hline Cowardin et al., (1979). & $\begin{array}{l}\text { Terrenos de transición entre sistemas acuáticos y terrestres donde } \\
\text { usualmente el nivel freático está en o cerca de la superficie, o el } \\
\text { terreno está cubierto por aguas someras. Los humedales deben tener } \\
\text { uno o más de los siguientes atributos: a) al menos periódicamente el } \\
\text { terreno alberga predominantemente hidrófilas; b) el sustrato } \\
\text { dominante corresponde a suelos hídricos y c) el sustrato está saturado } \\
\text { con agua o cubierto por aguas someras en algún momento de la } \\
\text { estación de crecimiento de cada año }\end{array}$ \\
\hline $\begin{array}{l}\text { Ministerio de Relaciones } \\
\text { Exteriores Chile. (2017) }\end{array}$ & $\begin{array}{l}\text { Las zonas húmedas que dividen en áreas de ciénagas, pantanos, áreas } \\
\text { de musgos o agua, sean estas naturales o artificiales, permanentes o } \\
\text { temporales, de aguas estáticas o corrientes, frescas, con helechos o } \\
\text { saladas, incluyendo zonas de agua de mar cuya profundidad no } \\
\text { exceda de seis metros durante la marea baja. }\end{array}$ \\
\hline $\begin{array}{l}\text { Fariña, J.M., y Camaño, A. } \\
\text { (2012). }\end{array}$ & $\begin{array}{l}\text { Ecosistemas asociados a sustratos saturados temporal o } \\
\text { permanentemente de agua, los cuales permiten la existencia y } \\
\text { desarrollo de biota acuática. }\end{array}$ \\
\hline $\begin{array}{l}\text { Portillo, A. \& Murillo J.M. } \\
\text { (2014). }\end{array}$ & $\begin{array}{l}\text { Los humedales son zonas de transición entre los ecosistemas } \\
\text { terrestres y acuáticos y poseen baja profundidad y suelen encontrarse } \\
\text { en llanuras inundadas muy próximos a los cursos de agua como los } \\
\text { ríos o lagos que les proveen el líquido. Algunos humedales están } \\
\text { conectados con aguas subterráneas. }\end{array}$ \\
\hline $\begin{array}{l}\text { Barbier, Acreman \& Knowler } \\
\text { (1997). }\end{array}$ & $\begin{array}{l}\text { Los humedales son ecosistemas cuya característica es la presencia de } \\
\text { agua durante periodos prolongados como para alterar los suelos, su }\end{array}$ \\
\hline
\end{tabular}




\begin{tabular}{|l|l|}
\hline & $\begin{array}{l}\text { flora y fauna, de tal forma que el suelo no actúa como en los hábitats } \\
\text { acuáticos o terrestres. }\end{array}$ \\
\hline
\end{tabular}

Fuente: Elaboración propia

Con el levantamiento de esta información bibliográfica y documental se han observado finalmente diferentes concepciones a nivel nacional e internacional sobre los humedales y sus principales características y, asimismo, se han podido analizar las normativas legales sobre la conservación de los ecosistemas y el patrimonio natural de estas zonas húmedas. Llama la atención, en este sentido, que el artículo 411 de la Constitución Ecuatoriana manifieste expresamente que el Estado garantizará la conservación, recuperación y manejo integral de los recursos hídricos, cuencas y caudales ecológicos asociados al ciclo hidrológico, por loque esta Carta Magna ya toma como base la definición más generalizada de la Convención Ramsar al formar parte Ecuador de este tratado internacional desde 1991.

Por otra parte, hemos de destacar también que el sistema de clasificación de paisajes de humedales se ha construido partiendo de las características generales de estos espacios en cuanto a formación y génesis, hidrología, geomorfología y protección de las aves migratorias, pero resulta llamativo que apenas se hayan considerado en la mayor parte de los casos los impactos antrópicos, las características socioculturales y las prácticas tradicionales de aprovechamiento de sus suelos y entornos.

De acuerdo con las características biológicas y físicas, la convención de Ramsar (2012) clasifica en 30 categorías a los humedales naturales y en otras 9 a los artificiales. Generalmente se reconocen cinco sistemas principales:

- Marino: humedales costeros incluyendo costas rocosas y arrecifes de coral.

- Estuarino: incluye deltas, marismas de marea y manglar.

- Lacustre: referente a lagos.

- Ribereño: humedales asociados a ríos y arroyos.

- Palustre: implica lodazales, marismas, pantanos y ciénagas.

A continuación, se presenta una síntesis de las clasificaciones establecidas por Ramsar en la Tabla 3.

Tabla 3. Tipo de humedales.

\begin{tabular}{|l|l|}
\hline $\begin{array}{l}\text { TIPO DE } \\
\text { HUMEDAL }\end{array}$ & DEFINICIÓN \\
\hline Marinos9 & Son humedales situados en las costas. \\
\hline Estuarinos & $\begin{array}{l}\text { Ubicados donde los ríos desembocan en el mar. Incluyen manglares, } \\
\text { deltas y marismas de mareas, cuya salinidad es media. }\end{array}$ \\
\hline Lacustres & Evidentemente, son humedales conectados con lagos. \\
\hline Palustres & Son humedales de aspecto pantanoso, como las marismas y las ciénagas. \\
\hline Ribereños & Están situados a los lados de ríos y arroyos. \\
\hline
\end{tabular}




\begin{tabular}{|l|l|}
\hline Artificiales & $\begin{array}{l}\text { Son creados por el hombre con un propósito específico: servir como } \\
\text { embalses, como criaderos de especies acuáticas comerciales, como } \\
\text { canales, etcétera. }\end{array}$ \\
\hline
\end{tabular}

Fuente: Elaboración propia

De acuerdo con la Convención Ramsar (2012) en Ecuador existen varios tipos de Humedales como resultado de la diversidad biológica y geográfica, de manera que podemos categorizarlos en tres grandes grupos en función de las casuísticas de las regiones geográficas del país. A continuación, se aporta un cuadro resumen en el que se muestran las categorías y los tipos de humedales (Tabla 4).

Tabla 4. Categorías y tipos de humedales en Ecuador

\begin{tabular}{|c|c|}
\hline CATEGORÍAS & TIPO \\
\hline Humedales marinos y costeros & 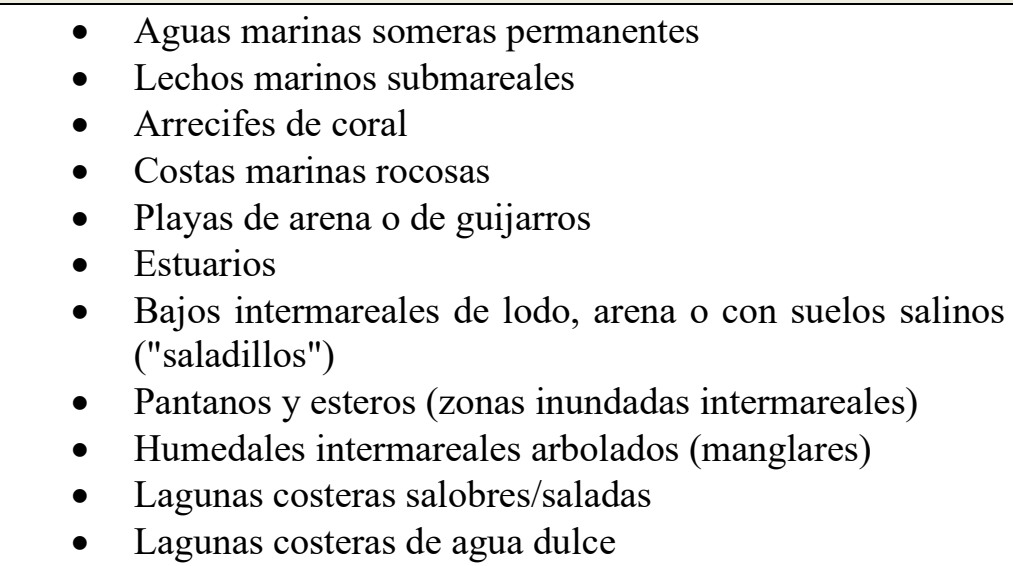 \\
\hline Humedales continentales & 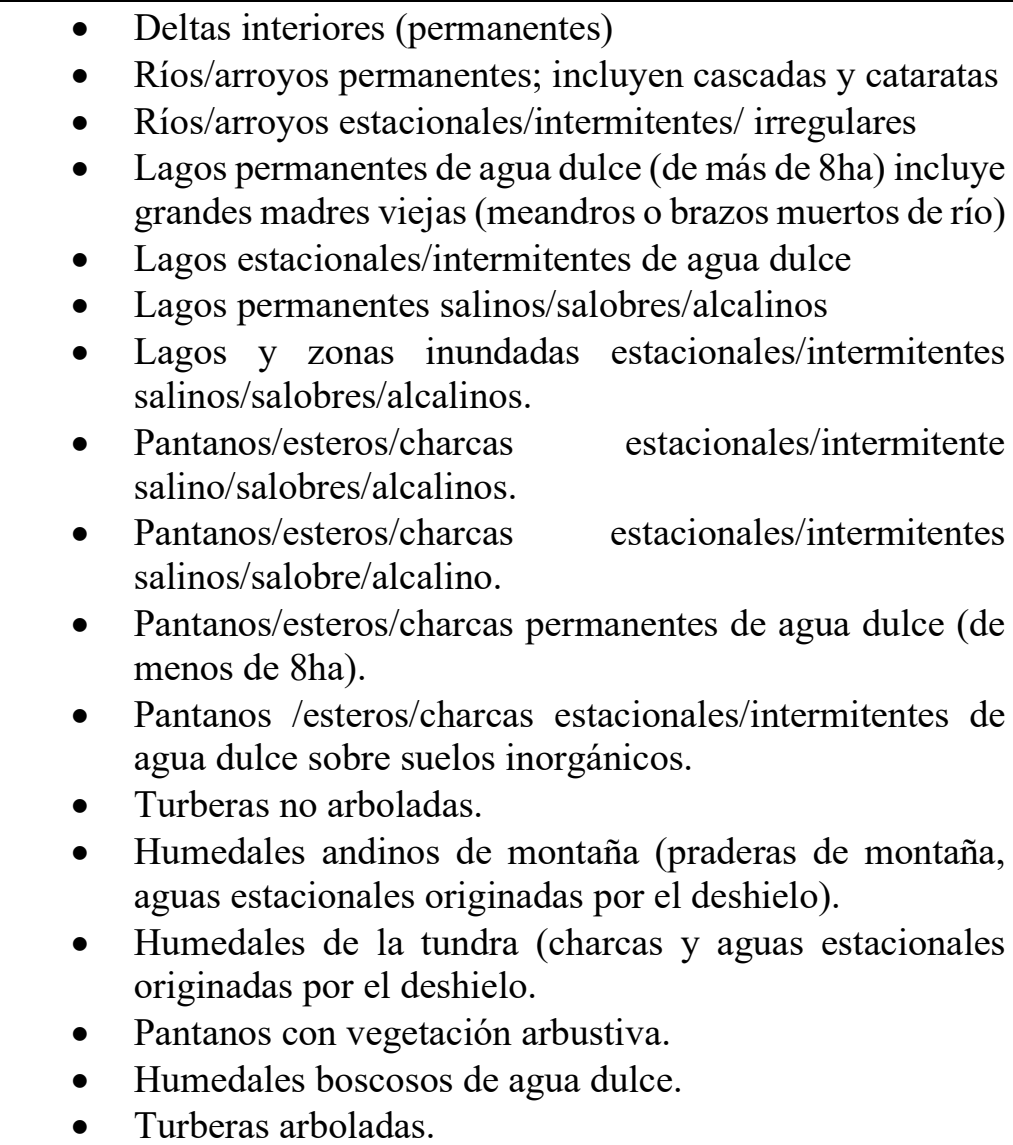 \\
\hline
\end{tabular}




\begin{tabular}{|c|c|}
\hline & $\begin{array}{l}\text { - Y. Manantiales de agua dulce, oasis. } \\
\text { - Humedales geotérmicos. } \\
\text { - Sistemas Kársticos y otros sistemas hídricos subterráneos } \\
\text { continentales. }\end{array}$ \\
\hline Humedales artificiales & 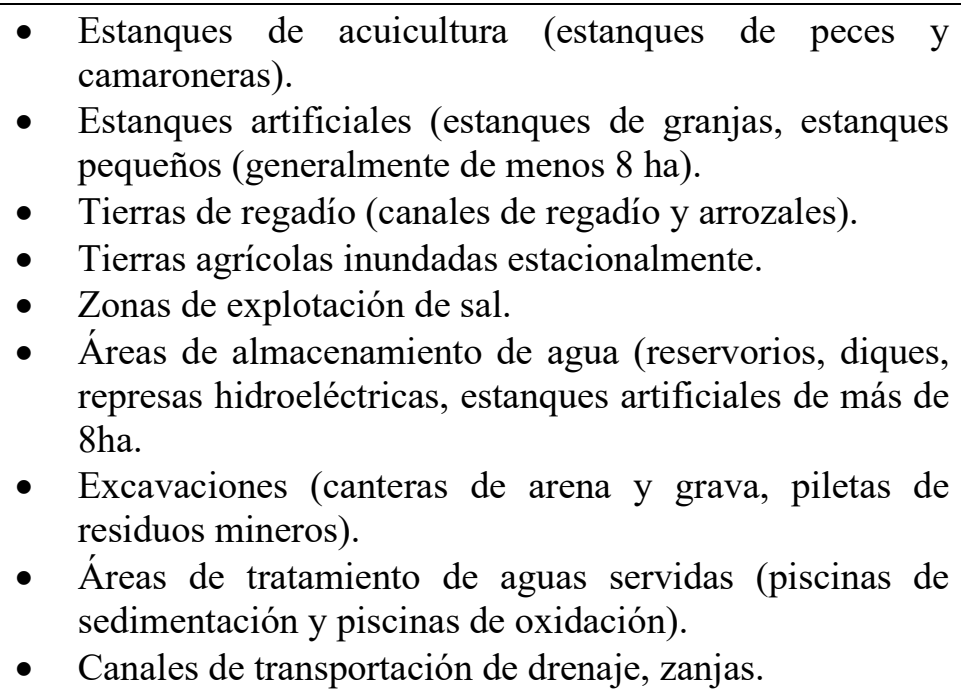 \\
\hline
\end{tabular}

Fuente: Elaboración propia

El conocimiento de las características, diferencias territoriales y clasificaciones de los humedales en Ecuador resulta fundamental para proceder a su posterior planificación y ordenación de los usos del suelo, evitando sus problemas actuales de deterioro y regresión. En este sentido, a lo largo del territorio ecuatoriano existen diferentes tipos de humedales en función de su geomorfología, pisos climáticos y biodiversidad, cuya caracterización y diferenciación recogemos en la Tabla 5 siguiente, de acuerdo con las principales investigaciones y estudios de caso analizados.

Tabla 5. Clasificación y definiciones tipológicas de los humedales de Ecuador

\begin{tabular}{|l|l|l|}
\hline AUTORES & TIPO DE HUMEDAL & REGIÓN \\
\hline $\begin{array}{l}\text { 1- Llambí y Cuesta (2014). } \\
\text { 2- Convención de Ramsar y } \\
\text { Grupo de Contacto EHAA. } \\
2008 .\end{array}$ & $\begin{array}{l}\text { Paramo: 1-Ecosistema propio de alta montaña, } \\
\text { entre los 3400 a 4500msnm, caracterizado por } \\
\text { vegetación de gramíneas, plantas en } \\
\text { almohadón, arbustos achaparrados y árboles } \\
\text { resistentes a la sequía fisiológica, congelación } \\
\text { del agua y suelo, baja presión de oxígeno, y }\end{array}$ & \\
donde ocurren precipitaciones en forma de & \\
lluvia, granizo o nieve. \\
2-Bioregión de las altas montañas tropicales \\
(Andes y centroamericana), se caracteriza por \\
una baja temperatura, pronunciadas variaciones \\
climáticas. La matriz vegetal es pajonal y sobre \\
ella se desarrollan comunidades complejas de \\
plantas con forma de roseta, arbustos y \\
bambúes, así como anegados cojines, musgos o \\
de plantas vasculares.
\end{tabular}




\begin{tabular}{|c|c|c|}
\hline $\begin{array}{l}\text { Podwojewski y Poulenard. } \\
(2000) .\end{array}$ & $\begin{array}{l}\text { Turba: formada por restos de plantas muertas y } \\
\text { parcialmente descompuestas que se han } \\
\text { acumulado in situ en terrenos anegados, son } \\
\text { ecosistemas que contribuyen a la diversidad } \\
\text { biológica, al ciclo hídrico mundial, al } \\
\text { almacenamiento mundial de carbono guardando } \\
\text { relación con el cambio climático y otras } \\
\text { funciones relativas a los humedales. }\end{array}$ & Andes/Amazonía \\
\hline Diva. (2000) & $\begin{array}{l}\text { Lago: gran masa de agua en extensas } \\
\text { hondonadas de terreno. Pertenecen a la } \\
\text { categoría de aguas quietas o lenticas. }\end{array}$ & Andes/Amazonía \\
\hline Roldán, (2008) & $\begin{array}{l}\text { Laguna: extensión de agua de menores } \\
\text { dimensiones que el lago. Extensión de agua } \\
\text { rodeada de tierra por todas partes. }\end{array}$ & Andes/Amazonía \\
\hline $\begin{array}{l}\text { Lima da Costa, M., Silva, y } \\
\text { Behling, H. (2005) }\end{array}$ & $\begin{array}{l}\text { Ciénaga: planicie inundada parcialmente o en } \\
\text { su totalidad, a modo de depósitos de aguas no } \\
\text { corrientes (ecosistemas lénticos), con algún } \\
\text { grado de conexión con el río, del cual depende } \\
\text { la renovación de sus aguas e intercambio de } \\
\text { materiales (sedimentos, solutos, coloides) y } \\
\text { organismos (plancton, bentos, propágulos y } \\
\text { plantas vasculares, juveniles y adultos de } \\
\text { invertebrados y peces). }\end{array}$ & Costa/Amazonía \\
\hline $\begin{array}{l}\text { Macintosh, D.J., Phillips, } \\
\text { M.J., Lewis, R.R. and } \\
\text { Clough, B. Review. (2002) }\end{array}$ & $\begin{array}{l}\text { Manglares: complejo de humedales } \\
\text { influenciados por las aguas polihalinas } \\
\text { provenientes de las mareas, el cual consiste en } \\
\text { bosques de manglar, playones mareales dentro } \\
\text { de la zona intermareal de latitudes tropicales y } \\
\text { subtropicales. Crecen en zonas costeras, } \\
\text { planicies o playas lodosas, fangosas o } \\
\text { cenagosas. }\end{array}$ & Costa \\
\hline Echevarría, H. (2008). & $\begin{array}{l}\text { Delta: depósito de aluviones fluviales formado } \\
\text { en la desembocadura de ríos en el mar. Terreno } \\
\text { comprendido entre los brazos de los ríos en la } \\
\text { desembocadura. }\end{array}$ & Costa \\
\hline Convención Ramsar. (2012). & $\begin{array}{l}\text { Estuario: son ecosistemas marino-costeros que } \\
\text { se ubican en la desembocadura de un río hacia } \\
\text { el mar. Se caracterizan por la presencia de } \\
\text { manglares, que son árboles muy tolerantes a los } \\
\text { cambios bruscos de salinidad. }\end{array}$ & Costa \\
\hline $\begin{array}{l}\text { CPPS/UNESCO/CI/Hivos. } \\
\text { (2016). }\end{array}$ & $\begin{array}{l}\text { Arrecifes de coral: son ecosistemas de alta } \\
\text { diversidad formados por corales resultado de } \\
\text { simbiosis entre pólipos y microalgas, los cuales } \\
\text { obtienen energía y alimento a través de la } \\
\text { fotosíntesis. Son medios ecosistémicos marinos } \\
\text { con una gran variedad de hábitats, diversidad de } \\
\text { especies y procesos biológicos. Estructuras } \\
\text { submarinas complejas, formadas por material } \\
\text { calcáreo (carbonato de calcio), producido por } \\
\text { los corales. }\end{array}$ & Costa \\
\hline $\begin{array}{l}\text { Mora, J.A. y Ramírez, N.A. } \\
\text { (2019). }\end{array}$ & $\begin{array}{l}\text { Estanques de acuicultura: Un estanque es un } \\
\text { depósito cerrado de agua, sin corrientes, de un } \\
\text { tamaño tal que puede ser utilizado para la } \\
\text { explotación de organismos acuáticos, }\end{array}$ & $\begin{array}{l}\text { Andes, } \\
\text { Amazonía, Costa }\end{array}$ \\
\hline
\end{tabular}




\begin{tabular}{|c|c|c|}
\hline & $\begin{array}{l}\text { incluyendo peces, moluscos, crustáceos y } \\
\text { plantas acuáticas. Son ecosistemas artificiales } \\
\text { manejando arrozales y otras instalaciones } \\
\text { construidas sobre tierra firme. }\end{array}$ & \\
\hline $\begin{array}{l}\text { Borja, A., Basset, A., } \\
\text { Bricker, S., Dauvin, J., Elliot, } \\
\text { M., Harrison, T., Marques, } \\
\text { J., Weisberg, S. \& West, R. } \\
\text { (2012). }\end{array}$ & $\begin{array}{l}\text { Tierras de regadío/o inundadas para } \\
\text { agricultura: zonas con propósitos de } \\
\text { explotación agrícola, son inundados temporal o } \\
\text { permanentemente mediante métodos artificiales } \\
\text { o naturales, en la mayoría el nivel freático es } \\
\text { superficial, se encuentran cercanos a fuentes } \\
\text { hídricas, la cultura más conocida es el arroz, se } \\
\text { crean ecosistema que generan alimentos para la } \\
\text { avifauna. }\end{array}$ & $\begin{array}{l}\text { Andes, } \\
\text { Amazonía, Costa. }\end{array}$ \\
\hline $\begin{array}{l}\text { Cowardin, L. M., V. Carter, } \\
\text { F. C. Golet and E. T. LaRoe } \\
\text { (1979) }\end{array}$ & $\begin{array}{l}\text { Áreas de almacenamiento de agua: presas, } \\
\text { represas, embalses que tienen la misma función } \\
\text { como ecosistemas artificiales, estructuras } \\
\text { edificadas con cualquier material, que } \\
\text { atraviesan un cauce natural o artificial, para } \\
\text { producir una elevación del nivel del agua o una } \\
\text { acumulación (embalse) y generar una } \\
\text { distribución de caudales diferente a la natural o } \\
\text { inicial. Se corresponden con ecosistemas } \\
\text { lénticos. Su principal característica es que son } \\
\text { una masa de agua sin corriente que puede } \\
\text { asemejarse a un lago o laguna. }\end{array}$ & $\begin{array}{l}\text { Andes, } \\
\text { Amazonía, Costa. }\end{array}$ \\
\hline
\end{tabular}

Fuente: Elaboración propia

En definitiva, los tipos más conocidos y comunes de humedales en Ecuador han podido ser identificados según regiones geográficas del país, lo que constituye un paso importante para su valorización, planificación y gestión, tanto ambiental como turístico-recreativa, más aún en la situación actual en que la mayoría de los ciudadanos y agentes locales que actúan en las áreas de influencia de estos espacios apenas relacionan a los humedales con las tipologías y conceptualizaciones sistematizadas en la Tabla 5, y más aún ni tan siquiera son conscientes de la importancia y la contribución que sus recursos hídricos tienen para el bienestar de las comunidades locales aledañas; de aquí, como hemos dicho antes, que este desconocimiento y falta de sensibilización favorezcan el deterioro y desaparición de los humedales, pese que un uso adecuado y una planificación estratégica de éstos fortalecería sectores productivos como el turismo, la producción agropecuaria, la pesca y la caza y, en general, el desarrollo sostenible de no pocos territorios vinculados a estos humedales.

Como hemos podido comprobar en la revisión bibliográfica practicada, debido a que los humedales son ecosistemas de transición entre ambientes acuáticos y terrestres, no hay una definición universalmente reconocida o comúnmente aceptada. Siendo esto así no cabe duda que se pueden generar confusiones y algunas situaciones inconsistentes a la hora de la clasificación, inventario y manejo sostenible de estas zonas húmedas y su propia valorización turístico-recreativa. Pero creemos, no obstante, que la definición de la Convención Ramsar puede aglutinar aceptablemente la mayor parte de las definiciones y conceptualizadas analizadas, como así sostienen no pocos autores, de manera que podríamos considerarla como oficial, más aun considerando que la mayoría de los países la están adoptando por formar parte del tratado internacional. $\mathrm{Y}$ esto sin perjuicio de que cada región o nación tenga sus particularidades geográficas y ecosistémicas y puedan permitirse el establecimiento de 
definiciones y clasificaciones más detalladas de acuerdo con sus características y particularidades.

La definición oficial de humedales en Ecuador es, de hecho, la propuesta por la Convención Internacional Ramsar, como consecuencia de los tratados internacionales vigentes que forman parte de su ordenamiento jurídico y de las normas de interpretación jurídica del Código Civil ecuatoriano. A esta definición oficial hemos de añadir y resaltar la caracterización constitucional de los humedales como ecosistemas frágiles y altamente sensibles en la normativa constitucional ecuatoriana.

\section{Conclusiones}

La investigación realizada en este trabajo, como resultado de la revisión bibliográfica sistemática, ha podido acercarnos a las diferentes definiciones, conceptualizaciones y caracterizaciones sobre las zonas húmedas, tanto las adoptadas a nivel general como las más detalladas y adaptadas a las casuísticas territoriales específicas de algunas regiones o zonas, en función de diversas variables y factores y las distintas interpretaciones de los autores y organismos consultados.

Debido a que los humedales representan ecosistemas de transición entre ambientes acuáticos y terrestres, se ha concluido en la revisión bibliográfica que no hay una definición universalmente reconocida o comúnmente aceptada, lo que ha conducido en no pocos casos a la generación de confusiones e incongruencias a la hora del tratamiento de estos espacios naturales en materia de planificación y gestión o el caso de la percepción de las potencialidades turístico-recreativas de estos humedales y su compatibilización con la conservación de sus ecosistemas y medio natural asociado.

Es evidente que una de las principales causas de los problemas ambientales y de deterioro de los humedales es el desconocimiento de la importancia de los servicios ecosistémicos y socio-productivos que estos aportan a la sociedad. Aunque se ha podido comprobar que se han dado avances en la conceptualización, caracterización y clasificación de los humedales, lo cierto es que éstos son aún insuficientes, al menos en lo que se refiere a la determinación de algunos criterios científicos para caracterizar las zonas húmedas en inventarios y sistemas de clasificación y analizar con más propiedad los procesos morfodinámicos, biogeográficos y de hábitats para la fauna y la flora, ciertamente complejos, que se dan cita en estos espacios.

Como hemos referido en el apartado anterior, la definición oficial de humedales en Ecuador es la propuesta por la Convención Internacional Ramsar, ya que en el país los tratados internacionales vigentes forman parte de su ordenamiento jurídico. Esta definición y su clasificación general correspondiente puede servir de paraguas y contenedor de otras clasificaciones y conceptualizaciones más detalladas y adaptadas a las casuísticas territoriales de las distintas regiones de Ecuador o de otras zonas a nivel mundial, como también de las diversas acepciones y caracterizaciones de los diferentes autores y organismos consultados.

En la revisión bibliográfica realizada, y particularmente en lo que se refiere a los humedales de Ecuador, se echan en falta estudios sobre el desarrollo socioeconómico de estos territorios y sus potencialidades para diversas actividades económicas como el turismo y la recreación y sus niveles de compatibilización con la conservación de sus ecosistemas de acuerdo con principios básicos de sostenibilidad. La caracterización socioambiental de estos espacios se revela como fundamental, así como el análisis de sus servicios ecosistémicos, que 
abarcan una serie de beneficios muy importantes para las comunidades locales aledañas, como es el caso de la producción de alimentos y la obtención de agua para el consumo humano. Todos estos análisis habrían de fomentarse con carácter general en todo el país y con carácter previo a la adopción de planes de manejo y ordenación de usos del suelo que pueda poner en marcha el Ministerio del Ambiente junto con otras administraciones de carácter regional, provincial o local, siendo también necesaria la caracterización socioambiental para la puesta en valor turístico-recreativo de estos espacios, que exigiría por añadidura una coordinación y colaboración conjunta entre la administración ambiental y la sectorial turística, cuyas competencias se están sustantivando en la actualidad de manera compartimentada y desconexionada en estos espacios.

Los humedales de Ecuador cuentan con suficientes recursos y entidad territorial como para albergar actividades tan diversas como la agricultura y el turismo, que en realidad pueden no solo compatibilizarse sino también conjugarse de forma sostenible para dar lugar a productos de agroturismo, ecoturismo y turismo ornitológico, sobre todo, además de diversas actividades de educación ambiental. Pero lamentablemente los gestores de estos espacios no poseen una visión holística, sino sesgada desde el punto de vista exclusivo de la conservación ambiental, de manera que manejen de forma separada e inadecuada, como si se trataran de compartimentos estancos, las distintas piezas territoriales y usos del suelo de las zonas húmedas, incluido el turísticorecreativo. Hay que considerar que una parte importante de los destinos turísticos más apreciados de Ecuador se ubican en áreas insulares, costeras y litorales que cuentan con ecosistemas de humedales que frecuentemente se conforman como un recurso territorial y un factor de atracción ciertamente importantes para los visitantes que se acercan a dichos destinos. Sin embargo, se tienen que tomar medidas como el control de visitantes, además de maximizar los beneficios que el turismo pueda aportar para que reviertan éstos tanto para la conservación del medio natural como para el desarrollo local.

\section{Financiación}

Este estudio es un resultado parcial de un proyecto I+D+I de la Escuela Superior Politécnica Agropecuaria de Manabí (ESPAM MFL), en Ecuador, que tiene como finalidad fundamentar desde el punto de vista teórico la conservación y el manejo sostenible de los humedales con categoría "Ramsar" de este país.

\section{Referencias}

Asamblea Nacional Constituyente del Ecuador (2008). Constitución de la República del Ecuador. Registro Oficial 449.

Asamblea Nacional Constituyente del Ecuador (2009). Ley Orgánica de la Biodiversidad de la República del Ecuador. Registro Oficial .449.

Asamblea Nacional Constituyente del Ecuador (2014). Ley Orgánica de recursos hídricos de la República del Ecuador. Registro Oficial .449.

Alfonso, A. y Dipotet, P. (2009). Propuesta de Clasificación de los humedales de la Provincia de Matanza. Matanzas, Cuba: Memorias del VIII Simposio Internacional de sobre Manejo de Humedales, CITMA, Unidad de Medio Ambiente de Matanzas.

Barbier, E.B.; Acreman, M., \& Knowler, D. (1997). Valoración económica de los humedales. Guía para decisores y planificadores. Gland, Suiza: Oficina de la Convención de Ramsar.

Borja, A., Basset, A., Bricker, S., Dauvin, J., Elliot, M., Harrison, T., Marques, J., Weisberg, S. \& West, R. (2012). Classifying ecological quality and integrity of estuaries. In E. Wolanski 
\& D. (Dir.). Treatise on Estuarine and Coastal Science (pp. 125-162). Waltham: Academic Press.

Cole, S. (2017). Water worries: An intersectional feminist political ecology of tourism and water in Labuan Bajo. Annals of Tourism Research, 67, 14-24.

CPPS/UNESCO/CI/Hivos. (2016). Plan de acción regional para la conservación de los manglares en el Pacífico Sudeste. Guayaquil, Ecuador: CPPS, UNESCO, Conservación Internacional e Hivos.

Convención de Ramsar y Grupo de Contacto EHAA. (2008). Estrategia Regional para la Conservación y Uso Sostenible de Humedales Altoandinos. Quito: Gobiernos de Ecuador y Chile, CONDESAN y TNC-Chile.

Convención Ramsar (2012). Definición de "humedales" y Sistema de Clasificación de Tipos de Humedales de la Convención de Ramsar. Recuperado de: http://archive.ramsar.org/cda/es/ramsar-documents-guidelines-classificationsystem/main/ramsar/1-31-105\%5E21235 40002

Cowardin, L. M., V. Carter, F. C. Golet and E. T. LaRoe (1979). Classification of wetlands and deepwater habitats of the United States, U.S. Washington, D.C.: Department of the Interior, Fish and Wildlife Service.

Chaoyang Fang, Zhanghua Tao, Dan Gao \& Hao Wu. (2016). Wetland mapping and wetland temporal dynamic analysis in the Nanjishan wetland using Gaofen One data. Annals of GIS, 22 (4), 259-271

De Groot, R. S., Alkemade, R., Braat, L., Hein, L., \& Willemen, L. (2010). Challenges in integrating the concept of ecosystem services and values in landscape planning, management and decision making. Ecological Complexity, 7(3), 260-272.

Diva, O. (2000). La gente y la biodiversidad. Centro para la Investigación de la Diversidad Cultural y Biológica de los Bosques Pluviales Andinos (DIVA), Dinamarca y Ediciones Abya Yala, Ecuador.

Doumet, Y. y Rivera, M. (2018). El turismo como instrumento para la conservación y desarrollo sostenible de los humedales en Ecuador: fundamentos jurídicos y teóricos para su planificación y gestión. Polo del Conocimiento, 24 (3), 53-84.

Do, Y., Kim, S.-B., Kim, J. Y., \& Joo, G.-J. (2015). Wetland-based tourism in South Korea: who, when, and why. Wetlands Ecology and Management, 23(4), 779-787.

Echevarría, H. (2008). Convención Ramsar en el Ecuador. Guía sobre la conservación y uso racional de los humedales. Quito, Ecuador: Centro Ecuatoriano de Derecho Ambiental.

Fariña, J.M., y Camaño, A. (2012). Humedales costeros de Chile. Aportes cientificos a su gestión sustentable. Santiago: Ediciones Universidad Católica de Chile.

Keddy, P. A. (2004). Wetland Ecology. Cambridge: Cambridge University Press,

Llambí, L.D. y Cuesta, F. (2014). La diversidad de los páramos andinos en el espacio y en el tiempo. En: Cuesta, F. et. Al. (Dir.). Avances en investigación para la conservación de los páramos andinos (pp. 7-40). Faculty FNWI: Institute for Biodiversity and Ecosystem Dynamics (IBED), FNWI: Institute for Biodiversity and Ecosystem Dynamics (IBED).

Martín M., Bravo J., Sandoval L., Biamonte E., Criado J. (2010). Conservación de los Humedales y Bienestar Humano en Centroamérica. Costa Rica: SEO/BirdLife, FUNGAP y Unión de Ornitólogos de Costa Rica. 
Martina, E. H.; Ryan, R.J.: Perry, J.; Hardin, A.; Rehema A.S.; Eustace, A. (2019). Assessing changes in Tanzania's Kwakuchinja Wildlife Corridor using multitemporal satellite imagery and open source tools. Applied Geography, 110. https://doi.org/10.1016/j.apgeog.2019.102051

Macintosh, D.J., Phillips, M.J., Lewis, R.R. and Clough, B. Review. (2002). Coastal Wetland Habitats and Shrimp Aquaculture. Washington: World Bank, NACA, WWF and FAO.

Ministerio de Ambiente Ecuador MAE. (2013). Manual para la Gestión Operativa de las Áreas Protegidas de Ecuador. Ecuador: Imprenta Mariscal.

Ministerio de Relaciones Exteriores Chile. (2017). Decreto $N^{\circ} 771$ de 1981. Promulga la Convención sobre zonas húmedas de importancia internacional especialmente como hábitat de las aves acuáticas, suscrito en Irán el 2 de febrero de 1971. Disponible en: http://bcn.cl/21lq4.

Minoo H. Esfehani \& Julia N. Albrecht. (2018). Roles of intangible cultural heritage in tourism in natural protected areas. Journal of Heritage Tourism, 13 (1), 15-29.

Mora, J.A. y Ramírez, N.A. (2019). Potencialidad del aviturismo para el desarrollo de iniciativas comunitarias en Cumaral Meta (Colombia). Revista Internacional de Turismo, Empresa y Territorio, 3 (2), 84-112.

Nunes, C.; Fernández, M.T. y Junk, W.J. (2014). Classificação e delineamento das Áreas Úmidas Brasileiras. Brasil: UFMT.

Lima da Costa, M., Silva, y Behling, H. (2005). Mineralogía e Geoquímica de sedimentos lacustres com substrato laterítico na Amazonia Brasileira. Revista Brasileira de Geociencias. 35(2): 176-171.

Llambí, L.D. y Cuesta, F. (2014). La diversidad de los páramos andinos en el espacio y en el tiempo. Avances en investigación para la conservación de los páramos andinos. Quito: CONDESAN.

Mulero Mendigorrri, A. y Rivera Mateos, M. (2018). Turismo de naturaleza y espacios naturales protegidos en España. Abaco: Revista de cultura y ciencias sociales, 98 (Ejemplar dedicado a: El turismo. Desarrollo, transformación y controversia de un fenómeno social), 8496.

Ooi, Esther Duke \& Joseph O'Leary. (2018). Tourism in changing natural environments. Tourism Geographies, 20:2, 193-201.

Park, E., Lee, S.J., Peters, D.J. (2017). Iowa wetlands outdoor recreation visitors' decisionmaking process: an extended model of goal-directed behavior. Outdoor Recreat. Tourism, 17, 64-76.

Polk, M. H., Young, K. R., Baraer, M., Mark, B. G., McKenzie, J. M., Bury, J., \& Carey, M. (2017). Exploring hydrologic connections between tropical mountain wetlands and glacier recession in Peru's Cordillera Blanca. Applied Geography, 78, 94-103.

Podwojewski y Poulenard. (2000). Los suelos de los páramos del Ecuador. En los suelos del páramo. Quito-Ecuador: GTP/Abya Yala..

Portillo, A. \& Murillo J.M. (2014). Identification of wetland water sources for environmental flow assessment: a case study of the Miguel Ibáñez wetlands (Segovia, Spain). Hydrological Sciences Journal, 59 (3-4), 466-487.

Rivera Mateos, M. (2010). Los centros de educación ambiental y su inserción en la oferta turística y recreativa de los espacios naturales protegidos: una aproximación desde Andalucía. En: López, D. (dir.). Turismo y gestión de espacios protegidos: XII Congreso Internacional de 
Turismo Universidad y Empresa (pp. 337-368). Castellón: Universidad Jaume I y Edit. Tirant lo Blanch.

Roldan, G. y Ramírez, J. (2008). Fundamentos de Limnología tropical, Bogotá: Editorial Universidad de Antioquía.

Secretaría de la Convención Ramsar. (2016). An Introduction to the Convention on Wetlands. Switzerland: Ramsar Convention Secretariat.

Secretaría de la Convención de Ramsar, (2010). Manejo de humedales: Marcos para manejar Humedales de Importancia Internacional y otros humedales. Manuales Ramsar para el uso racional de los humedales. Gland (Suiza): Secretaría de la Convención de Ramsar.

Secretaría de la Convención Ramsar. (2013). Definición de "humedales" y Sistema de Clasificación mde Tipos de Humedales de la Convención de Ramsar. Recuperado en: http://archive.ramsar.org/cda/es/ramsar-documents-guidelines-

classificationsystem/main/ramsar/1-31-105\%5E21235 4000 _

Spence, C.; Guan, X.J. y Phillips, R. (2011). The Hydrological Functions of a Boreal Wetland. Wetlands, 31, 75-85.

Songjun Xu, Hyun Jeong Kim, Mingzhu Liang \& Kisang Ryu. (2018). Interrelationships between tourist involvement, tourist experience, and environmentally responsible behavior: a case study of Nansha Wetland Park, China, Journal of Travel \& Tourism Marketing, 35 (7). https://doi.org/10.1080/10548408.2018.1439429

Woodcock, T.S.; Monaghan, M.C. y Alexander, K. (2010). Ecosystem Characteristics and Summer Secondary Production in Stormwater Ponds and Reference Wetlands. Wetlands, 30, 461-474.

Xiaofei Yua, Mingju Eb, Mingyang Suna,c, Zhenshan Xuea, Xianguo Lua, Ming Jianga, Yuanchun Zou. (2018). Wetland recreational agriculture: Balancing wetland conservation and agrodevelopment. Environmental Science and Policy, 87, 11-17. 